\title{
The Kinematic Modeling and Simulation of Rotating-Contact Jujube Harvest Method
}

\author{
Hailong He ${ }^{1, a}$, Wei Zhang ${ }^{1,2, b}$, Dong Chen ${ }^{1, c}$ and Zheng Jin ${ }^{1,2, d}$ \\ ${ }^{1}$ Beijing Forestry Machinery Research Institute of the State Forestry Administration, 100029, Beijing, China \\ ${ }^{2}$ Forestry New Technology Research Institute, Chinese Academy of Forestry, 100091, Beijing, China

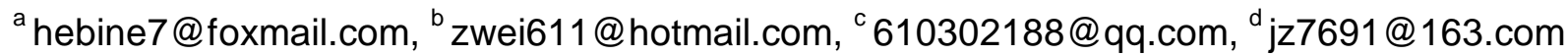

\begin{abstract}
Keywords: Mechanized harvesting, Trajectory equation, Parameter optimization, MATLAB
Abstract. Jujube is a type of forest-fruit product originated in China. Its harvest mainly depends on manual picking resulting in low efficiency, high strength and cost. In order to improve the level of mechanization of harvesting jujube, this research investigated a rotating-contact harvest method of jujube. Based on the working principle of harvest, the kinematic model was established and parametric equations of the trajectory were deduced. According to the MATLAB numerical analysis software, the effect of structural parameters on the trajectory was investigated and suitable structural parameters of jujube harvest were obtained. The results showed that kinematic model and parametric equations were reasonable which could be applied to jujube harvest. The influence of the change in parameter $\beta$ on the motion trajectory was most significant and the structural parameters for jujube harvest were that $\beta$ was $\pi / 36$, $\mathrm{r}$ was $200 \mathrm{~mm}$ and $\mathrm{h}$ was $200 \mathrm{~mm}$. Based on the optimization of structural parameters, the harvest method could also be applied to harvest other fruits. The results from this research can provide a theoretical basis for the design and development of harvest equipment.
\end{abstract}

\section{Introduction}

As a type of forest-fruit product originated in China, jujube is mainly distributed over the five provinces, including Shanxi, Hebei, Shandong, Henan and Shanxi, and the Xinjiang Uygur Autonomous Region. With the development of domestic economy and the increase in demand for jujube fruit, the planting area and yield of jujube trees in China are gradually increasing $[1,2]$. However, the harvest of jujube mainly depends on manual picking due to the complex site conditions, restricting the operation of large machinery. Considering the low productivity and high labor intensity of manual picking as well as the rising labor costs, it is urgently needed to develop a suitable jujube harvest device to promote the further development of jujube industry.

In recent years, many studies related to forest-fruit harvesting machines were conducted around the world. Various types of practical harvesting devices were developed and solved some of the existing difficulties in fruit harvesting [3-7]. Deboli et al. designed a handheld olive harvester and obtained its acceleration data by simulation the vibration response of olive branches, which were used to analyze the impact of vibration transmission to operators [8,9]. Erdogan et al. developed a type of inertial vibration machine to harvest apricot and optimized its harvest frequency, amplitude and time through experiments [10]. Torregrosa et al. developed a type of orange harvesting machine, experimented it on oranges of different varieties from different producing areas and acquired the data of harvest rate, separating force and damage $[11,12]$. Guo et al. developed a traction vibrating blueberry harvest machine targeting high and low-bush plants $[13,14]$. Du et al. developed a stroke-adjustable monodirectional pulling forest-fruit harvesting machine [15]. Li et al. developed mechanical arms and harvesting machines for camellia [16,17].

To the best of our knowledge, there is a lack of sufficient information concerning machines of harvesting jujube. Jujube is rarely cultivated in other countries except for China in the world. This results in the lack of related research except for the 4YS-24 Jujube Harvest Machine invented by the mechanical equipment research institute of Xinjiang agricultural reclamation science academy. It was employed together with tractors, whereas restricted by the diverse growing environments of jujube, so it was unable to enter some of the orchards. Furthermore, over-shaking the tree trunks could easily 
result in the death of jujube trees $[18,19]$. So far, mechanical harvest methods included pneumatic, shaking, percussive, and contacting [4]. Considering some disadvantages of the shaking methods, this research investigated a rotating-contact method used to harvest jujube. Its working principles were also discussed and a corresponding kinematics model was established. Based on MATLAB data analyzing software, the influence of various parameters on motion trajectory was studied to obtain the suitable motion parameters for jujube harvesting. The results from this research will provide a theoretical foundation for design and development of model machines.

\section{The Model}

The Working Principles of Rotating-Contact Harvest Method. The contacting harvest method usually involves stretching a vibrator with many thin sticks directly into the canopy and using different vibration modes. As the harvest method directly touches the fruits or the side branches near the fruits, it doesn't need strong vibrating force and leading to less damage to the fruit trees. According to the varying vibration modes, it could mainly be classified into reciprocating, rotating and hybrid [4]. The reciprocating type of motion requires relatively larger operation space. Furthermore, its mode of motion continuously adds and reduces speed with low efficiency and high energy-consuming. The hybrid type of motion requires more complex equipment structures and relatively larger sizes. The rotating type of motion, on the other hand, is consistent and efficient, in which the fast rotation with small amplitude could strike the side branches and fruits effectively. Therefore, the rotating-contact method is employed to harvest jujube.

Considering the jujube's growing environment, the rotating-contact method is required to operate in small space, easily move in the canopy, and have small vibration amplitude so as to reduce the harm for fruits and branches [20,21]. Meanwhile, it also needs to have a simple design, conveniently operation and low cost, so as to be popularized among the vast majority of fruit growers. The working principles of the rotating-contact method are illustrated in Fig. 1, in which A-axis, B-axis and C-axis intersected at point $\mathrm{O}$, and in the middle of $\mathrm{A}$-axis is an oblique axis with the intersection angle $\beta$. On the oblique axis, there attached a shaft sleeve which could oscillate around B-axis, and the shaft sleeve together with the plane $\mathrm{B}$-axis is in could oscillate around $\mathrm{C}$-axis. When power source is inputted and drives A-axis to rotate, the shaft sleeve, driven by the oblique axis, will oscillate around B-axis and $\mathrm{C}$-axis simultaneously. In this way, the shaft sleeve's mode of motion is a composition of oscillation in two vertical directions, which forms a motion trajectory of a close loop curve in space. Through attaching suitable comb structures to the shaft sleeve, this mode of motion could be outputted to harvest jujube fruits.

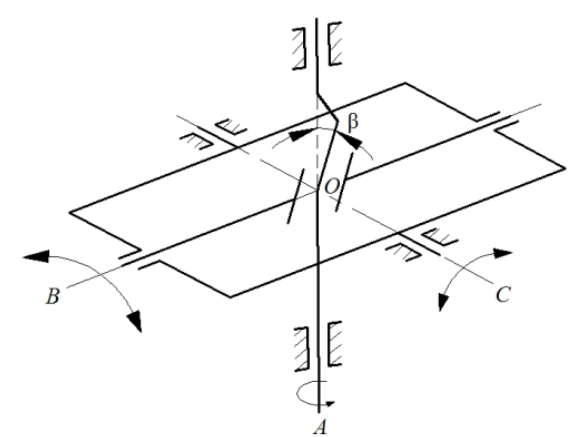

Fig. 1. Illustration of the working principles of rotating-contact harvest method

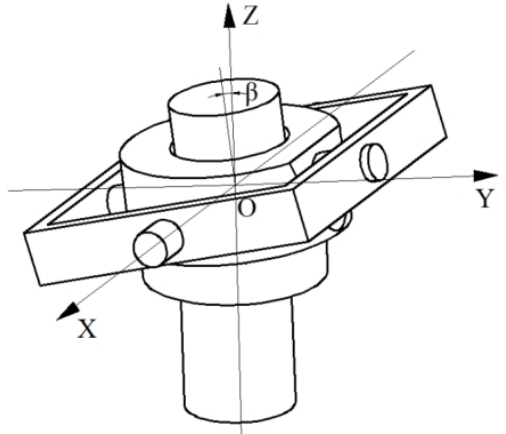

Fig. 2. A brief diagram of the kinematics model of rotating-contact harvest method

Kinematics Modeling. To study the motion trajectory of rotating-contact harvest method, a kinematics model of the transmission system is established to obtain the specific parameter equations of the output motion. As the motion of harvesting operation is outputted by attaching suitable comb structure to the shaft sleeve, the end of the comb structure could be abstracted as a point on the shaft sleeve enlarged in radius and height, which means every point on the comb structure can correspond to 
a size of shaft sleeve. In this way, the motion trajectory of rotating-contact harvest method is converted into solving the motion trajectory of a point on the shaft sleeve. Establish a Space Cartesian coordinate system, with point $\mathrm{O}$ as the origin, the direction of $\mathrm{C}$-axis as $\mathrm{X}$-axis, the direction of $\mathrm{A}$-axis as $\mathrm{Z}$-axis, and the direction vertical to both $\mathrm{A}$-axis and $\mathrm{C}$-axis as $\mathrm{Y}$-axis. The brief diagram of the kinematics model is shown in Fig. 2.

Since the mode of motion of the shaft sleeve is oscillating around B-axis while oscillating around $\mathrm{C}$-axis together with the plane $\mathrm{B}$-axis is in, and the direction of $\mathrm{C}$-axis is the direction of $\mathrm{X}$-axis, the motion of the shaft sleeve could be separated into the motion in plane XOZ and the motion in plane YOZ. The motion trajectory equations of the shaft sleeve could be obtained from the simultaneous parameter equations of the motions in the two planes.

First, the motion in plane YOZ is investigated and the motion in the direction of $\mathrm{X}$-axis is ignored. For the convenience of analysis, the kinematics model is further simplified by taking an end point on the generatrix formed by the shaft sleeve intersecting with plane YOZ as the study object, showed in Fig. 3. Driven by A-axis, the shaft sleeve's position moves from the solid line to the dotted line in half a cycle, that is, from point 1 to point 1'. Thus, studying the motion trajectory of point 1 could give the parameter equations of the motion of the shaft sleeve in plane YOZ.

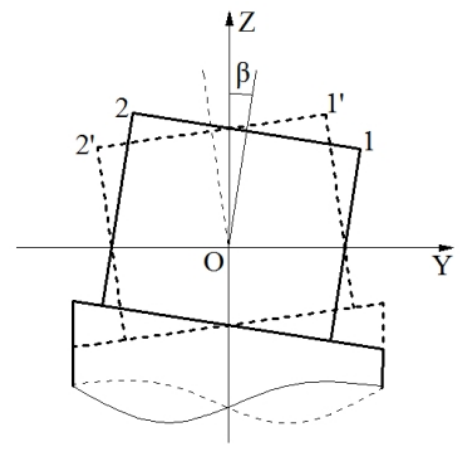

Fig. 3.The schematic diagram of motion in the plane YOZ

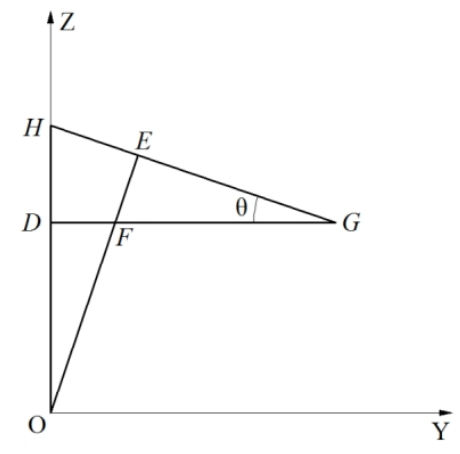

Fig. 4. Diagram of the geometric relationship of motions in the plane YOZ

In order to obtain the motion equations of point 1 in plane YOZ, it needs to establish the coordinate relationship of point 1 . Referring to the schematic diagram of point 1's motion inside plane YOZ, we can extract the corresponding geometric relationship, showed in Fig. 4. Then, acquiring the coordinate of point 1 is converted into solving the length of DG and OD.

Where, EG is the radius of the cylinder shaft sleeve point $1, \mathrm{OE}$ is the distance between the origin $\mathrm{O}$ and the upper surface of the cylinder shaft sleeve, and $\theta$ is the intersection angle between Y-axis and the intersection line of the upper surface of the cylinder shaft sleeve and plane YOZ. Let $E G=r, O E=h$, then it can be gotten:

$$
\begin{aligned}
& \mathrm{EF}=\mathrm{r} \cdot \tan \theta \\
& \mathrm{FG}=\mathrm{r} / \cos \theta \\
& \mathrm{OF}=\mathrm{OE}-\mathrm{EF}=\mathrm{h}-\mathrm{r} \cdot \tan \theta . \\
& \mathrm{DF}=\mathrm{OF} \cdot \sin \theta=h \cdot \sin \theta-\mathrm{r} \cdot \sin \theta \cdot \tan \theta . \\
& \mathrm{OD}=\mathrm{CF} \cdot \cos \theta=h \cdot \cos \theta-\mathrm{r} \cdot \sin \theta . \\
& \mathrm{DG}=\mathrm{DF}+\mathrm{FG}=\mathrm{h} \cdot \sin \theta-\mathrm{r} \cdot \sin \theta \cdot \tan \theta+\mathrm{r} / \cos \theta=h \cdot \sin \theta+r \cdot \cos \theta .
\end{aligned}
$$


Given that during the motion process of the cylinder shaft sleeve, the intersection angle between $\mathrm{Y}$-axis and the intersection line of the upper surface of the cylinder shaft sleeve and plane YOZ is constantly changing, in order to determine the variation trend of $\theta$, it needs to establish the relationship between the intersection angle $\theta$ and the rotating angle $\sigma$ of A-axis, which in turn leads to the relationship between the intersection angle $\theta$ and the rotating speed $\mathrm{n}$. The change of the intersection angle $\theta$ with the rotation of A-axis could be simplified into the change of the intersection angle between the connection line of one point on the oblique plane with the axis and the bottom surface during the process of an oblique cylinder rotating around its own axis, as shown in Fig. 5.

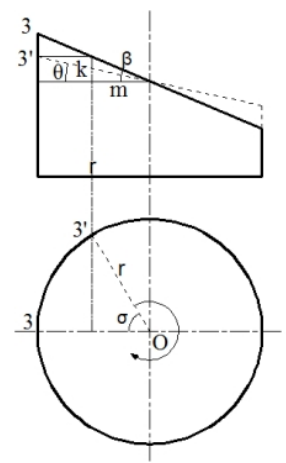

Fig. 5. The relationship between the intersection angle $\theta$ and the rotating angle $\sigma$ of A-axis

Where, $\beta$ is the inclination angle of the oblique section, which is the inclination angle of the middle oblique axis of A-axis, and $\sigma$ is the angle that A-axis has passed in its rotation. For point 3 on the oblique section, when A-axis rotates $\sigma$ angle, its projection on the bottom plane will move from point 3 to point 3', which means its height reduced from the height of point 3 to the height of point 3' in the oblique plane. As a result, the intersection angle between the oblique section and the horizontal plane changes from the initial angle $\beta$ to the current angle $\theta$. Then it can be gotten:

$$
\begin{aligned}
& \mathrm{m}=\mathrm{r} \cdot \cos \sigma \\
& \mathrm{k}=\mathrm{m} \cdot \tan \beta=\mathrm{r} \cdot \cos \sigma \cdot \tan \beta \\
& \tan \theta=\mathrm{k} / \mathrm{r}=\cos \sigma \cdot \tan \beta \\
& \theta=\arctan (\cos \sigma \cdot \tan \beta)
\end{aligned}
$$

In this way, it could obtain the formula for the relationship between the intersection angle $\beta$ and the rotating angle of A-axis $\sigma$ and also the initial angle $\beta$. The motion equations in the YOZ plane are as follows:

$$
\begin{aligned}
& Y=D G=h \cdot \sin \theta+r \cdot \cos \theta=h \cdot \sin (\arctan (\cos \sigma \cdot \tan \beta))+r \cdot \cos (\arctan (\cos \sigma \cdot \tan \beta)) \\
& Z=O D=h \cdot \cos \theta-r \cdot \sin \theta=h \cdot \cos (\arctan (\cos \sigma \cdot \tan \beta))-r \cdot \sin (\arctan (\cos \sigma \cdot \tan \beta))
\end{aligned}
$$

In order to obtain the changes of the coordinates in the direction of $\mathrm{X}$-axis, as well as establishing the motion relationships of $\mathrm{X}$-axis correspond with $\mathrm{Y}$-axis and $\mathrm{Z}$-axis, it needs to investigate the movements of point 1 in plane XYZ as described above. When point 1 moves to the position of point 1' in plane $\mathrm{XYZ}$, its coordinate on $\mathrm{X}$-axis is always zero, which is a special case and can only be considered as a verification of the equations at the later stage. However, through adjusting the position of point 1 ahead by $1 / 4$ cycle, i.e. ahead by $\pi / 2$ phase in terms of the rotating angle $\sigma$, the position relationship of point 1 in plane $\mathrm{XOZ}$ is consistent with the position relationship of point 1 in plane 
YOZ. Thus, the $\mathrm{X}$-axis coordinate of point 1 could be expressed by its $\mathrm{Y}$-axis coordinate, with the difference of $\pi / 2$ in terms of rotating angle phase. It could be concluded that:

$$
X=h \cdot \sin (\arctan (\cos (\sigma-\pi / 2) \cdot \tan \beta))+r \cdot \cos (\arctan (\cos (\sigma-\pi / 2) \cdot \tan \beta)) \text {. }
$$

Based on the relationship between the rotating angle, the rotating speed and time $\sigma=\omega \cdot t=2 \pi \cdot n \cdot t$, we could obtain the motion equation of point 1 , which is the trajectory equation of the output movement of the shaft sleeve:

$$
\begin{aligned}
& X=h \cdot \sin (\arctan (\tan \beta \cdot \sin 2 \pi \cdot n \cdot t))+r \cdot \cos (\arctan (\tan \beta \cdot \sin 2 \pi \cdot n \cdot t)) . \\
& Y=h \cdot \sin (\arctan (\tan \beta \cdot \cos 2 \pi \cdot n \cdot t))+r \cdot \cos (\arctan (\tan \beta \cdot \cos 2 \pi \cdot n \cdot t)) . \\
& Z=h \cdot \cos (\arctan (\tan \beta \cdot \cos 2 \pi \cdot n \cdot t))-r \cdot \sin (\arctan (\tan \beta \cdot \cos 2 \pi \cdot n \cdot t)) .
\end{aligned}
$$

\section{Results and Discussion}

Simulation Analysis of Motion Trajectory. It was found from the motion equations that the motion trajectory was mainly affected by the parameters $h, r$, and $\beta$. It needed to determine the values of these three parameters in order to obtain the motion trajectory for jujube harvesting. Based on the specific working conditions and the overall requirements for the structure and size of harvesting equipment, the roughly estimated value ranges of the parameters were that $\mathrm{h}$ changed from $100 \mathrm{~mm}$ to $400 \mathrm{~mm}, \mathrm{r}$ changed from $100 \mathrm{~mm}$ to $400 \mathrm{~mm}$ and $\beta$ changed from $\pi / 60$ to $\pi / 12$. In order to obtain the influence of the main parameters on the motion trajectory, it needed to maintain the other parameters constant and change one parameter at a time, and conducted simulation analysis of the motion equations using MATLAB data analysis software. The simulation parameters were set as follows. In the first time, $h$ was $250 \mathrm{~mm}, \mathrm{r}$ was $250 \mathrm{~mm}$ and $\beta$ changed from $\pi / 60$ to $\pi / 12$. In the second time, $\mathrm{h}$ was $250 \mathrm{~mm}, \mathrm{r}$ changed from $100 \mathrm{~mm}$ to $400 \mathrm{~mm}$ and $\beta$ was $\pi / 20$. In the third time, h changed from $100 \mathrm{~mm}$ to $400 \mathrm{~mm}$, $\mathrm{r}$ was $250 \mathrm{~mm}$ and $\beta$ was $\pi / 20$. The tendency graphs of the influence of various parameters on the motion trajectory obtained by simulation were showed in Fig. 6-8.
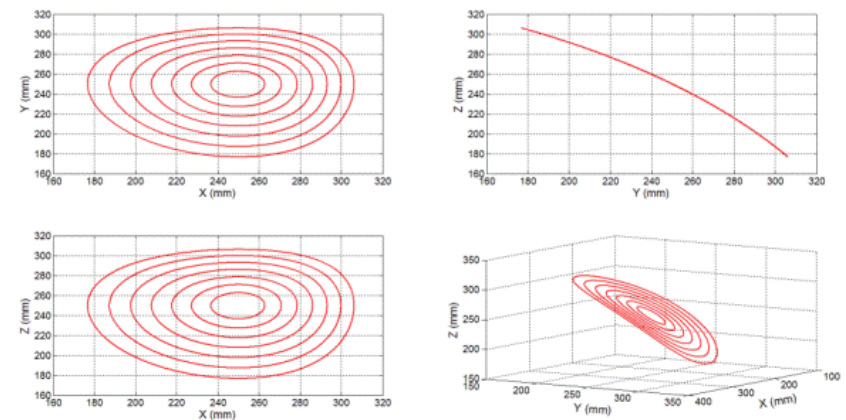

Fig. 6. The influence of the change in $\beta$ on the motion trajectory

According to Fig. 6, it was found that the change in $\beta$ significantly changed the motion trajectory in the directions of three coordinate axes. The projection dimensions of the motion trajectory on each coordinate axis increased with increase in $\beta$. Similarly, the covering area of the motion trajectory also increased with increase in $\beta$. The surface surrounded by the motion trajectory bend to a certain extent, and the inclination angle also underwent minor variation. Thus, the $\beta$ angle needed to be controlled in a certain range in order to reach the suitable inclination and amplitude. 

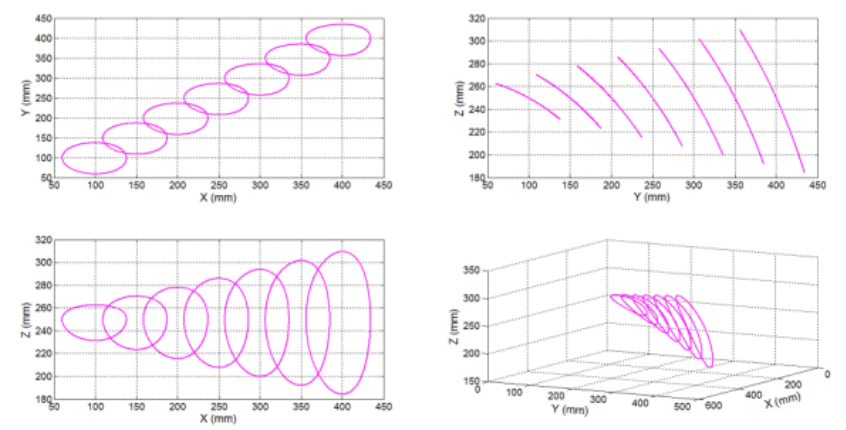

Fig. 7. The influence of the change in $r$ on the motion trajectory

According to Fig. 7 , it was found that the change in $r$ did not result in the change in motion trajectory in the directions of $\mathrm{X}$-axis and $\mathrm{Y}$-axis. The dimension of the motion trajectory in the direction of $\mathrm{Z}$-axis increased and the intersection angle of the motion trajectory plane and $\mathrm{Z}$-axis decreased with increase in $r$. Thus, the value of $r$ should be neither too large nor too small. It needed to maintain suitable inclination between the motion trajectory plane and Z-axis and also suitable amplitude in the direction of Z-axis in order to facilitate the harvesting operations.
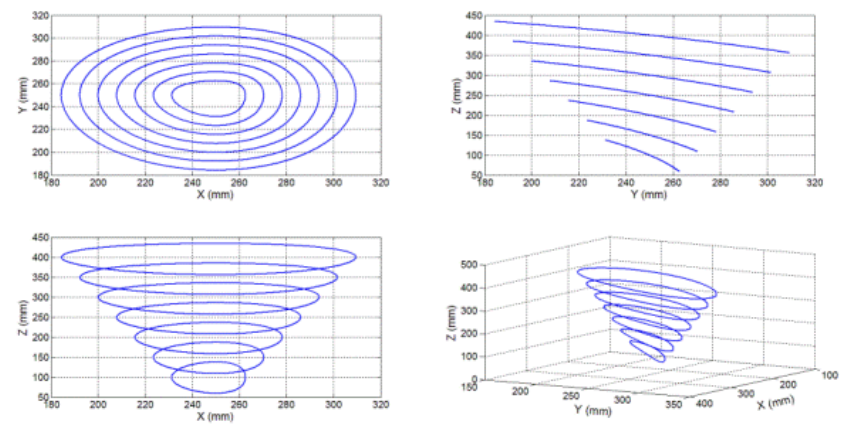

Fig. 8. The influence of the change in h on the motion trajectory

According to Fig. 8, it was found that the dimensions of the motion trajectory in the directions of $\mathrm{X}$-axis and $\mathrm{Y}$-axis increased with increase in $\mathrm{h}$. It was interesting that the dimension variation in the direction of Z-axis was not significant and the motion trajectory plane tended to become more horizontal. That is, the inclination gradually decreased. Thus, the value of $\mathrm{h}$ should not be set too high.

The Determination of Motion Trajectory Parameters. To achieve the best harvesting results, it needed to set the equipment parameters according to the growing conditions and characteristics of the harvesting target-jujube. Based on the actual observations of the growing state of the jujube tree branches, the size of the working head of the harvesting equipment should not be too large, and the operation space should not be too larger to avoid disturbing the fast movements of the equipment in harvesting operation. The space between the comb sticks of the comb should correspond to the average radius of the jujube fruits to ensure the fruits being struck off when passing through the comb sticks. According to relevant references [21,22], the average radius of jujube fruit is $30 \mathrm{~mm}$, so the space between comb sticks should also be set to $30 \mathrm{~mm}$. In order to ensure the harvesting operation space completely cover the space position the comb, the motion trajectory of two comb sticks should be tangent to each other. That is, the projection of the motion trajectory in the $\mathrm{X}$-axis direction should be no less than $30 \mathrm{~mm}$.

To determine the optimum value of parameter $\beta$, other parameters should be temporarily determined base on relevant harvesting experience. And then, the optimum parameter $\beta$ could be obtained through simulation analysis when the motion trajectory meets the requirements. According to Fig. 6, the amplitude also increased and the trajectory produces distortions to a certain extent with increase in $\beta$. The value of $\beta$ should therefore be appropriately set from $\pi / 60$ to $\pi / 18$. The value selection of $h$ and $r$ could determine the space position the output point of the motion trajectory, so the value of both parameters would subject to the restriction of the structure and size of equipment. According to experiences and requirements, h should be $200 \mathrm{~mm}$ and $\mathrm{r}$ should be $200 \mathrm{~mm}$. Using 
MATLAB data analysis software, the above parameters into the motion equations to conduct simulation were analyzed. Fig. 9 showed the impact on motion trajectory when parameter $\beta$ was from $\pi / 60$ to $\pi / 18$.
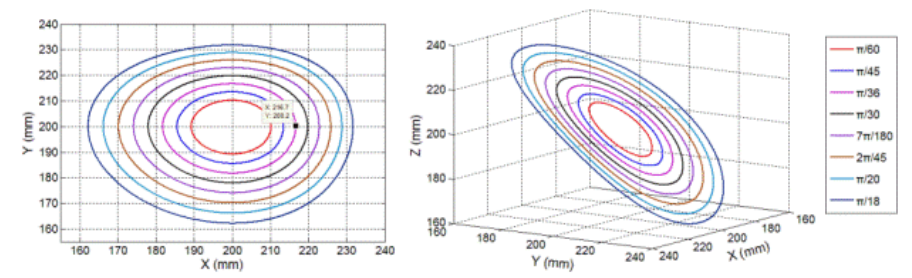

Fig. 9. Optimizing the impact of $\beta$ on motion trajectory

According to Fig. 9, it was found that the trajectory whose projection on X-axis direction was no less than or close to $30 \mathrm{~mm}$ was the motion trajectory when $\beta$ was $\pi / 36$, and the trajectory's projection dimensions on each coordinate axis were relatively similar, so that the speed would be stable in the rotating process and would not result in shock phenomenon. Furthermore, the inclination of the output motion trajectory plane was around 45 degree, which could enable the motion trajectory to contact with the fruits and branches in each direction to transmit the vibration excitation efficiently. As indicated by the simulation optimum results, when designing rotating-contact jujube harvesting method, it should firstly be set the inclination of the oblique axis $\beta$ of $\pi / 36$ among the parameter influencing the motion trajectory, and then set $\mathrm{h}$ of $200 \mathrm{~mm}$ and $\mathrm{r}$ of $200 \mathrm{~mm}$, and could have minor adjustments according to the actual situation.

\section{Conclusions}

This research designed a type of rotating-contact method to harvest jujube. Based on the working principle of harvest, the kinematic model was established and parametric equations of the trajectory were deduced. With fast rotation and small amplitude of comb sticks, this method could strike the side branches and the fruits effectively and reduce the harm to fruits and branches.

Based on MATLAB data analysis software and the extent of influence of the change in parameters $\beta, \mathrm{r}$ and $\mathrm{h}$ on the motion trajectory, simulation of the motion equations were gotten. The change in $\beta$ significantly influenced the projection dimensions of the motion trajectory in the directions of three coordinate axes. At the same time, the inclination of the motion trajectory plane also underwent change. But the impact of the change in $r$ and $h$ on the motion trajectory was not significant compared to $\beta$. According to relevant harvesting experience and requirements, the optimum parameters for jujube harvesting were that $\beta$ was $\pi / 36$, r was $200 \mathrm{~mm}$ and $\mathrm{h}$ was $200 \mathrm{~mm}$.

\section{Acknowledgements}

This work was financially supported by the Project 948 of the State Forestry Administration (2013-4-17).

\section{References}

[1] Y.H. Zhou, J.F. Bi, Q.Q. Chen, X. Liu, X.Y. Wu, R.J. Chen and C.L. Shao: Food Machinery. Vol. 29 (2013), p. 214-217

[2] X. Chen, Y.J. Li, Q. Yang, Y. Chen, Y.J. Wang, X.G. Weng, Q. Li, X.X. Zhu and X.C. Wang: World Science and Technology/Modernization of Traditional Chinese Medicine and Materia Medica. Vol. 17 (2014), p. 687-691

[3] W.H. Cao, J.Z. Wang, F. Huang, M.L. Yang and Y.X. Huang: Systems Engineering-Theory \& Practice. Vol. 35 (2015), p. 2857-2865 
[4] D. Chen, X.Q. Du, S.M. W and Q. Zhang: Transactions of the Chinese Society of Agricultural Engineering. Vol. 27 (2011), p. 195-200

[5] Z. Jin, G. Yang, W. Zhang, J.H. Yang and S.Y. Wang: Wood Processing Machinery. Vol. 26 (2015), p. 43-44+54

[6] S.N. Jha, K. Narsaiah, A.D. Sharma, M. Singh, S. Bansal and R. Kumar: J Food Sci Technol. Vol. 47 (2010), p. 1-14

[7] P.L. Li, S.H. Lee and H.Y. Hsua: Procedia Engineering. Vol. 23 (2011), p. 351-366

[8] R. Deboli, A. Calvo, C. Preti and M. Inserillo: International Journal of Industrial Ergonomics. Vol. 44 (2014), p. 581-589

[9] R. Deboli, A. Calvo, F. Gambella, C. Preti, R. Dau and E.C. Casu: Agric Eng Int: CIGR Journal. Vol. 16 (2014), p. 228-235

[10]D. Erdogan, M. Guner, E. Dursun and I. Gezer: Biosystems Engineering. Vol. 85 (2003), p. 19-28

[11]A. Torregrosa, E. Ortì, B. Martìn, J. Gil and C. Ortiz: Biosystems Engineering. Vol. 104 (2009), p. $18-24$

[12]C. Ortiz, J. Blasco, S. Balasch and A. Torregrosa: Biosystems Engineering. Vol. 110 (2011), p. $2-9$

[13] Y.L. Guo, Y.D. Bao, P.Z. He and H.B. Wang: Transactions of the Chinese Society of Agricultural Engineering. Vol. 28 (2012), p. 40-45

[14] L. Geng, Y.L. Guo and H.B. Wang: Transactions of the Chinese Society of Agricultural Machinery. Vol. 47 (2016), p. 1-9

[15] X.Q. Du, K.N. Ni and C.Y. Wu: Transactions of the Chinese Society of Agricultural Machinery. Vol. 47 (2016), p. 59-66

[16] Z.C. Gao, L.J. Li, X. Li, S.H. Min and C.F. Yi: Transactions of the Chinese Society of Agricultural Engineering. Vol. 29 (2013), p. 19-25

[17] S. Li, J. Zhou, L.J. Li, C.F. Yi, Z.C. Gao and S.S. Yang: Journal of Agricultural Mechanization Research. Vol. 37 (2015), p. 46-49

[18] H.Y. Yang, Z. Kan, L.H. Wang, W. Fu, R. He and Y. Sun: Journal of Agricultural Mechanization Research. Vol. 34 (2012), p. 77-80

[19] W. Fu, R. He, J.L. Qu, Y. Sun, L.H. Wang and Z. Kan: Journal of Agricultural Mechanization Research. Vol. 36 (2014), p. 106-109

[20] X.W. Fan, H. Zhang, S.H. Ma, C.F. Li, H.Z. Zhang and W. Meng: Journal of Tarim University. Vol. 25 (2013), p. 34-38

[21] C. Yan, P. Liu, M.J. Liu, J.R. Wang, D.C. Kong and D.K. Li: Scientia Silvae Sinicae. Vol. 46 (2010), p. 78-86

[22] P.T. Shi, H.X. Wen, and N.X. Wang: Transactions of the Chinese Society of Agricultural Engineering. Vol. 28 (2012), p. 28-34 